\title{
Immunization with Modified Vaccinia Virus Ankara-Based Recombinant Vaccine against Severe Acute Respiratory Syndrome Is Associated with Enhanced Hepatitis in Ferrets
}

\author{
Hana Weingartl, ${ }^{1,2} \dagger$ Markus Czub, ${ }^{2,3} \dagger$ Stefanie Czub, ${ }^{1,2}$ James Neufeld, ${ }^{1}$ Peter Marszal, ${ }^{1}$ Jason Gren, ${ }^{1}$ \\ Greg Smith, ${ }^{1}$ Shane Jones, ${ }^{3}$ Roxanne Proulx, ${ }^{3}$ Yvonne Deschambault, ${ }^{3}$ Elsie Grudeski, ${ }^{3}$ \\ Anton Andonov, ${ }^{2,3}$ Runtao He ${ }^{2,3}$ Yan Li, ${ }^{2,3}$ John Copps, ${ }^{1}$ Allen Grolla, ${ }^{3}$ \\ Daryl Dick, ${ }^{3}$ Jody Berry, ${ }^{1,2}$ Shelley Ganske, ${ }^{1}$ Lisa Manning, ${ }^{1}$ \\ and Jingxin $\mathrm{CaO}^{2,3 *}$ \\ National Centre for Foreign Animal Diseases ${ }^{1}$ and National Microbiology Laboratory, ${ }^{3}$ Canadian Science \\ Centre for Human and Animal Health, Winnipeg, Manitoba, Canada R3E 3R2, and Department \\ of Medical Microbiology, University of Manitoba, Winnipeg, \\ Manitoba, Canada R3T 2N2 2
}

Received 29 April 2004/Accepted 29 July 2004

\begin{abstract}
Severe acute respiratory syndrome (SARS) caused by a newly identified coronavirus (SARS-CoV) is a serious emerging human infectious disease. In this report, we immunized ferrets (Mustela putorius furo) with recombinant modified vaccinia virus Ankara (rMVA) expressing the SARS-CoV spike (S) protein. Immunized ferrets developed a more rapid and vigorous neutralizing antibody response than control animals after challenge with SARS-CoV; however, they also exhibited strong inflammatory responses in liver tissue. Inflammation in control animals exposed to SARS-CoV was relatively mild. Thus, our data suggest that vaccination with rMVA expressing SARS-CoV S protein is associated with enhanced hepatitis.
\end{abstract}

Severe acute respiratory syndrome (SARS) is a serious emerging human infectious disease of the 21st century. The causative agent was identified and characterized as a new member of the family Coronaviridae, SARS-associated coronavirus (SARS-CoV) $(8,14)$. The first SARS outbreak was primarily contained by the means of quarantine. As evidenced by the recently reported new cases (http://www.wpro.who.int/sars /docs/pressreleases/pr_27122003.asp and http://www.who.int/csr /don/2004_04-23/en/), it is almost certain that SARS-CoV remains a constant threat to public health. Efforts to develop SARS vaccine candidates are under way worldwide (9). Several recent reports have described studies on evaluation of SARS vaccine candidates in monkey and mouse models $(1,7,15,19)$. It has been shown that a recombinant adenovirus-based SARS vaccine candidate expressing SARS-CoV spike (S) and nucleocapsid proteins could induce strong neutralizing antibody and T-cell responses in monkeys (rhesus macaques) (7). However, the protection potential was not evaluated by challenge experiment. The neutralizing antibody response induced by recombinant modified vaccinia virus Ankara (rMVA) expressing SARS-CoV S protein (rMVA-S) has been shown to inhibit SARS-CoV replication in a mouse model (1). Since replication of SARS-CoV in mice can last only approximately 3 days postinfection, the memory immune response, which is essential for an effective prophylactic vaccine, is difficult to evaluate in

\footnotetext{
* Corresponding author. Mailing address: National Microbiology Laboratory, Canadian Science Centre for Human and Animal Health, 1015 Arlington St., Winnipeg, MB, Canada R3E 3R2. Phone: (204) 789-6052. Fax: (204) 789-2082. E-mail: jingxin_cao@hc-sc.gc.ca.

$\dagger$ H.W. and M.C. contributed equally to this work.
}

mice. In this communication, we evaluated the effects of rMVA-S in ferrets.

Coronavirus $\mathrm{S}$ is the major antigenic protein responsible for inducing neutralizing antibody responses $(4,6)$, while MVA is a widely used recombinant poxvirus vector for development of safe and effective recombinant vaccines (11). We constructed rMVA-S using a standard protocol for construction of recombinant poxviruses with the recombinant vector pJS5, provided by Bernard Moss at the National Institutes of Health $(2,5)$. The S gene was synthesized based on a SARS-CoV Tor2 isolate (8) by reverse transcription-PCR (RT-PCR) with primer pair AGGCGAATTCATGTTTATTTTCTTATTATTTCTTA CTCTCACT (N terminus primer; EcoRI site in italics) and TATACCCGGGTTATGTGTAATGTAATTTGACACCCT

TGAGAA (C terminus primer; SmaI site in italics). Expression of the $\mathrm{S}$ protein was confirmed with Western blot analysis using a specific monoclonal antibody against the SARS-CoV S protein (Fig. 1).

Since it has been reported that ferrets were susceptible to SARS-CoV infection (10), we decided to use ferrets for an immunization-and-challenge study. Animal housing and manipulations were approved by the Animal Care Committee of the Canadian Science Centre for Human and Animal Health and met the Canadian Council on Animal Care guidelines. Sixto 10 -week-old male (castrated) ferrets were purchased from Marshall Farm Pet Supplies (Wolcott, N.Y.). Enzyme-linked immunosorbent assay (ELISA) and neutralization tests were performed to confirm that there was no antibody cross-reactivity against SARS-CoV in all ferrets before the experiment. Ferrets were divided into three groups (see Tables 1 and 2; 


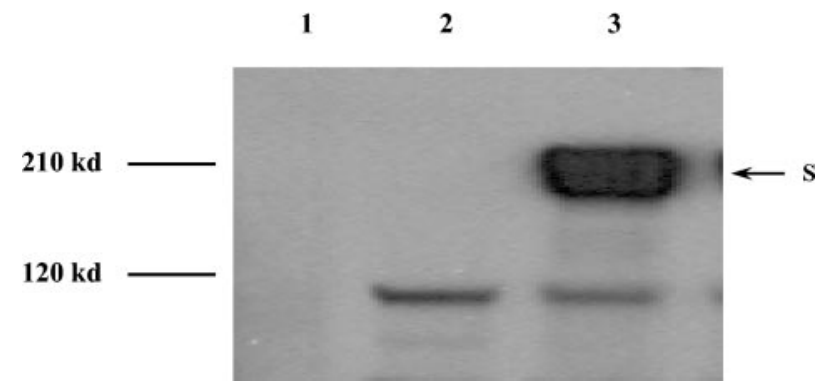

FIG. 1. Expression of SARS-CoV S proteins by rMVA-S. The $\mathrm{S}$ protein was detected by Western blotting with a SARS-CoV S-specific mouse monoclonal antibody (1). Lane 1, mock-infected BHK21 cell control; lane 2, lysate from MVA-infected BHK21 cells; lane 3, lysate from rMVA-S-infected BHK21 cells.

ferrets were housed individually, i.e., one ferret per cage) and were immunized with parental MVA (ferrets 1 to 3), rMVA-S (ferrets 7 to 9), or phosphate-buffered saline (PBS; ferrets 10 to 12 ) on day 0 with a dose of $10^{8} \mathrm{PFU}$ of the corresponding virus per ferret by intraperitoneal and subcutaneous routes, and a booster immunization was given on day 14 with the same regimen. Due to the lack of ferret-specific reagents for analysis of cell-mediated immune responses, only neutralizing antibody responses were monitored by microplaque reduction neutralization test. Briefly, a triplicate of serial dilutions of heatinactivated ferret sera were incubated with $100 \mathrm{PFU}$ of the SARS-CoV in a $100-\mu l$ volume for $60 \mathrm{~min}$ at $37^{\circ} \mathrm{C}$. After incubation, the serum-SARS-CoV mixture was added to monolayers of Vero-V76 cells in a 96-well tissue culture plate. Infected Vero-V76 cells were incubated at $37^{\circ} \mathrm{C}$ in $5 \% \mathrm{CO}_{2}$ for 3 to 4 days with a $2 \%$ carboxymethylcellulose overlay and then fixed with $4 \%$ formaldehyde and stained with $0.5 \%$ crystal violet. Neutralization titers were determined as the reciprocal of the highest serum dilution that neutralized at least $70 \%$ of the formation of plaques on Vero-V76 cells. As shown in Table 1 , neutralizing activity was detected in sera collected from all three ferrets 7 days after booster immunization with rMVA-S virus, while the titer declined to undetectable level 14 days after

TABLE 1. Neutralizing antibody response following rMVA-S immunization and SARS-CoV challenge

\begin{tabular}{|c|c|c|c|c|c|c|c|c|c|c|c|}
\hline \multirow{3}{*}{$\begin{array}{c}\text { Ferret } \\
\text { no. }\end{array}$} & \multirow{3}{*}{ Immunogen $^{a}$} & \multicolumn{10}{|c|}{ Neutralization titer on indicated day(s): } \\
\hline & & \multicolumn{5}{|c|}{ Postvaccination } & \multicolumn{5}{|c|}{ Postchallenge } \\
\hline & & 0 & 7 & $14^{b}$ & 21 & 28 & $3-5$ & $7-9$ & $13-15$ & $20-22$ & $27-29$ \\
\hline 1 & MVA & - & - & - & - & - & - & 320 & 320 & 160 & 320 \\
\hline 2 & MVA & - & - & - & 20 & - & - & 160 & 160 & 640 & 640 \\
\hline 3 & MVA & - & - & - & - & - & 40 & 160 & 320 & 640 & 640 \\
\hline 7 & rMVA-S & - & - & - & 40 & - & 20 & 1,280 & 1,280 & 640 & 640 \\
\hline 8 & rMVA-S & - & 20 & - & 40 & - & 80 & 1,280 & 640 & 640 & 1,280 \\
\hline 9 & rMVA-S & - & - & - & 20 & - & 640 & 2,560 & 320 & 1,280 & 1,280 \\
\hline 10 & PBS & - & - & - & - & - & - & 320 & 320 & 1,280 & 1,280 \\
\hline 11 & PBS & - & - & - & - & - & - & 320 & 320 & 640 & 1,280 \\
\hline 12 & PBS & - & - & - & - & - & 20 & 80 & 320 & 1,280 & 1,280 \\
\hline
\end{tabular}

\footnotetext{
${ }^{a}$ Immunogen MVA is the parental virus of rMVA-S.

${ }^{b}$ The day booster immunization was given; the neutralizing antibody response was determined by microplaque reduction neutralization test, and the lowest dilution used was $1 / 20$. - , negative.
}

the booster. The serum immunoglobulin $\mathrm{G}$ titer determined by ELISA corresponded with the neutralization results (see online supplementary data). Moreover, the antibody response specific for the $\mathrm{S}$ protein in rMVA-S-vaccinated ferrets was confirmed by Western blotting.

Two weeks after the booster immunization, ferrets were challenged with $10^{6} \mathrm{PFU}$ of the SARS-CoV Tor 2 isolate by the intranasal route in our biosafety level 4 facility. Ferrets immunized with rMVA-S developed a neutralizing antibody response as early as 3 days after SARS-CoV challenge, while neutralizing antibodies were not detected in most of the control ferrets until 7 days after SARS-CoV infection (Table 1). This shows that a typical memory immune response occurred in ferrets immunized with rMVA-S following SARS-CoV challenge. Furthermore, ferrets immunized with rMVA-S developed peak neutralizing antibody titer between 7 and 9 days after SARS-CoV challenge. In contrast, other challenged ferrets developed comparable levels of neutralizing antibodies 13 days later (Table 1). To our knowledge, this is the first report showing that immunization with a SARS vaccine candidate induced a rapid memory immune response, which is an essential feature for an effective prophylactic vaccine, following SARS-CoV challenge in an animal model.

Although no clinical signs (e.g., elevated temperature and altered behavior including feeding) were observed up to 29 days postchallenge, viral RNA was detected in pharyngeal swabs and blood samples by RT-PCR from all ferrets challenged with SARS-CoV by our previously reported protocols (17). The viral RNA could be detected in pharyngeal swabs starting from 1 day after SARS-CoV infection (Table 2). Ferret 8 (vaccinated with rMVA-S) still shed virus in the pharyngeal excretion up to 22 days postchallenge. Interestingly, viral RNA could be detected in blood specimens only at 8 days postchallenge and lasted up to 22 days postinfection. Moreover, live SARS-CoV could be isolated from pharyngeal swabs early in the infection (up to 5 days postinfection; level ranged from $4 \times 10^{3}$ to $1.4 \times 10^{4}$ PFU per pharyngeal swab, and no significant difference between rMVA-S-immunized and control ferrets was observed), while an attempt to isolate SARS$\mathrm{CoV}$ from sera was not successful (data not shown). We speculate that the failure to isolate live virus from pharyngeal swabs (5 days postinfection) and sera (8 days postinfection) is related to the increased neutralizing antibody response (Table 1$)$. Real-time RT-PCR to quantify the virus loads from the positive blood specimens (as determined by classical RT-PCR; Table 2) was also unsuccessful. The sensitivity of our real-time RT-PCR has been titrated to be $0.1 \mathrm{PFU}$ per $\mathrm{ml}$, while the classical RT-PCR showed sensitivity as low as $10^{-4}$ PFU per ml (A. Andonov and H. Weigartl, unpublished data). Thus, although SARS-CoV could enter into blood after approximately 8 days of challenge, viral load was low (lower than 0.1 PFU per ml). Nonetheless, our data indicate that immunization with rMVA-S had no significant effects on the level of SARS-CoV replication in ferrets, although a rapid, vigorous memory neutralizing antibody response occurred. In contrast, a live SARSCoV (15), a DNA-based vaccine expressing the S protein (19), and rMVA-S (1) have been reported to induce significant protective immunity in mice following SARS-CoV challenge. The most likely cause for the difference in the protective efficacy in mice and ferrets immunized with rMVA-S is that 
TABLE 2. Detection of viral RNA by RT-PCR from blood and pharyngeal swabs.

\begin{tabular}{|c|c|c|c|c|c|c|c|c|c|c|c|c|c|}
\hline \multirow{3}{*}{$\begin{array}{c}\text { Ferret } \\
\text { no. }\end{array}$} & \multirow{3}{*}{ Immunogen } & \multicolumn{12}{|c|}{ Presence of viral RNA ${ }^{a}$ on indicated days in: } \\
\hline & & \multicolumn{6}{|c|}{ Blood } & \multicolumn{6}{|c|}{ Pharyngeal swabs } \\
\hline & & $1-3$ & $4-6$ & $8-10$ & $13-15$ & $20-22$ & $27-29$ & $1-3$ & $4-6$ & $8-10$ & $13-15$ & $20-22$ & $27-29$ \\
\hline 1 & MVA & - & - & + & - & + & - & + & - & + & - & - & - \\
\hline 2 & MVA & - & - & - & - & + & - & + & + & + & - & - & - \\
\hline 3 & MVA & - & - & - & + & - & - & + & + & - & - & - & - \\
\hline 7 & rMVA-S & - & - & + & + & - & - & + & + & + & - & - & - \\
\hline 8 & rMVA-S & - & - & + & + & + & - & + & + & + & + & + & - \\
\hline 9 & rMVA-S & - & - & - & + & - & - & + & + & + & - & - & - \\
\hline 10 & PBS & - & - & + & + & - & - & + & + & - & - & - & - \\
\hline 11 & PBS & - & - & - & + & + & - & + & + & + & + & - & - \\
\hline 12 & PBS & - & - & - & - & - & - & + & + & + & - & - & - \\
\hline
\end{tabular}

${ }^{a}$ Due to the limited working capacity in a biosafety level 4 space, only one ferret from a group could be manipulated for blood and pharyngeal swab collection on a particular day after SARS-CoV challenge. +, RT-PCR positive; -, RT-PCR negative.

SARS-CoV exhibits different replication kinetics in these two animal systems. For example, SARS-CoV could replicate to a high titer in the first 3 days after challenge in mice, while the virus titer decreased sharply afterwards (15). Replication of SARS-CoV in ferrets, however, could last up to 22 days after infection (Table 2). Thus, further studies to elucidate the kinetics of SARS-CoV replication in ferrets should aid in under- standing the difference between these two animal models for development of SARS vaccines.

Biochemical tests of blood samples and histopathological examination of various tissues were performed to investigate any pathological effects as consequences of rMVA-S vaccination and SARS-CoV challenge. The VetTest dry chemistry analyzer, with the protocol and reagents provided by the man-
A

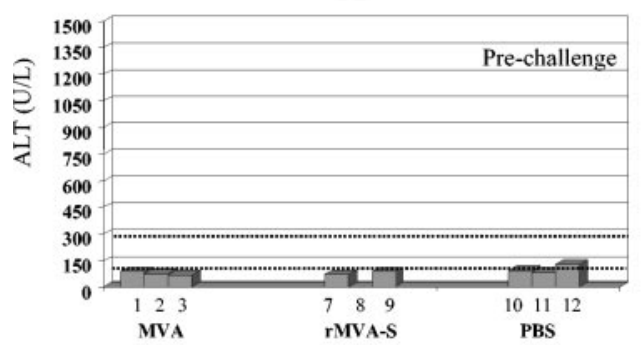

C

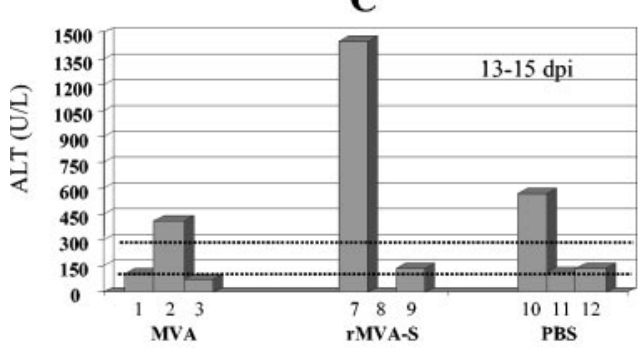

$\mathbf{E}$

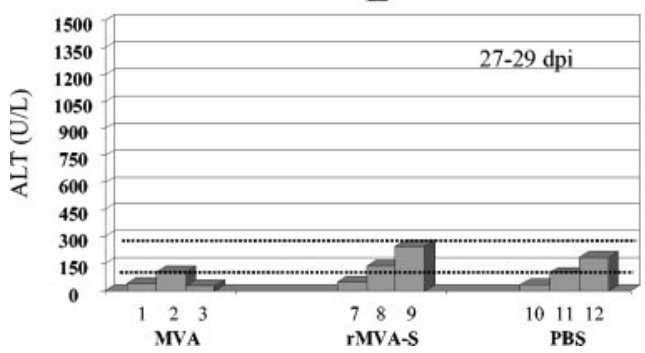

B

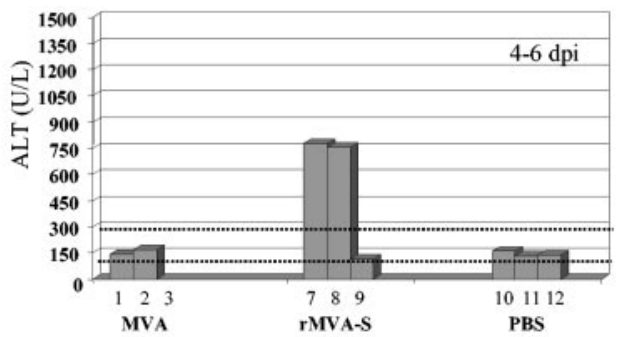

D

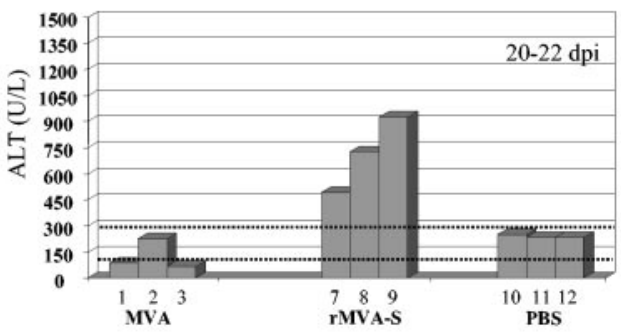

FIG. 2. ALT level following rMVA-S immunization and SARS$\mathrm{CoV}$ challenge. (A) Prechallenge (ferret 8 sample not available) sample; (B) sample taken 4 to 6 days after infection with SARS-CoV (ferret 3 sample not available); (C) sample taken 13 to 15 days postinfection (dpi; ferret 8 sample not available); (D) sample taken 20 to 22 dpi; (E) sample taken 27 to 29 dpi. ALT values between the dotted scale lines are considered normal reference values. 

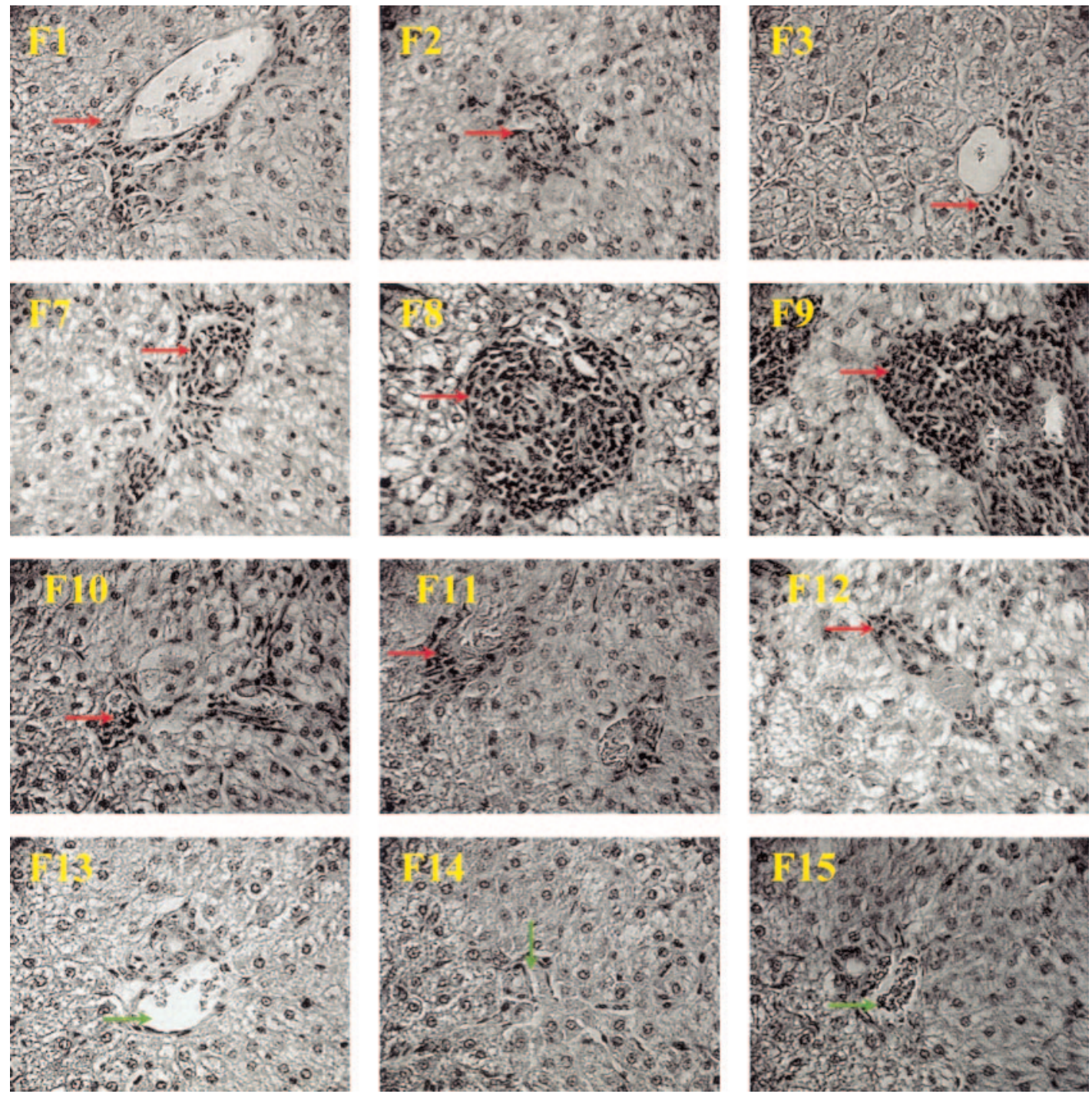

FIG. 3. Histopathology of ferret livers following immunization and SARS-CoV challenge. Representative pictures were taken at 27 to 29 days postinfection (ferrets [F] 1 to 3, 7 to 9, and 10 to 12) or no infection (naive ferrets 13,14, and 15; exposed to neither MVA nor SARS-CoV) at $\times 40$ magnification. Perivascular mononuclear infiltrates (red arrows) were present in all livers from ferrets infected with SARS-CoV, ranging from mild (ferrets 1, 2, 3, 10,11, and 12) to severe (ferrets 7, 8, and 9) lesions. In addition, intralobular infiltration of mononuclear cells was extensive in livers from ferrets 7, 8, and 9. No significant liver lesions were found in naive ferrets (ferrets 13, 14, and 15, which did not receive MVA or SARS-CoV and which were used for preliminary examination of ferrets. Green arrows, vein of portal triads.

ufacturer (IDEXX Laboratories Inc. USA), was used to examine blood samples taken at various time points for the levels of alkaline phosphatase (an indicator of hepatic disease involving the biliary system), alanine aminotransferase (ALT; an indicator of hepatic parenchymal lesions), albumin (an indicator of abnormality of hepatic and renal function), creatinine (an indicator of renal disease), total bilirubin (an indicator of obstructive liver disease), total protein (an indicator of abnormality of hepatic and renal function), and urea (an indicator of renal disease). Surprisingly, ferrets vaccinated with rMVA-S demonstrated a significantly higher level of ALT after challenge with SARS-CoV than the control ferrets (Fig. 2). The elevated level of ALT was evidenced by 4 to 6 days after SARS-CoV infection and lasted until day 22. All the other parameters tested fell into the normal or slightly higher-thannormal (alkaline phosphatase) physiological range compared to the reference value (based on the recommendation from IDEXX Laboratories Inc. USA and data from 60 serum samples from healthy ferrets provided by the ferret supplier, Marshall Farm).

Histopathological examination was performed on liver sections (fixed with 10\% PBS-buffered formalin, embedded in wax, and stained with hematoxylin and eosin) from ferrets sacrificed between 27 and 29 days postchallenge. It was found that ferrets immunized with rMVA-S (particularly ferret 9) developed severe periportal and panlobular mononuclear hepatitis (Fig. 3). In contrast, only mild periportal mononuclear hepatitis was observed in control ferrets receiving parental MVA or PBS. In addition, the panlobular hepatitis observed in rMVA-S-immunized ferrets was also accompanied by signs of focal necrosis of liver cells, including swelling of hepatocytes (hydropic degeneration), increased acidophilia, and hyper- 
chromatic and fragmented nuclei (karyorrhexis). Thus, the histopathological finding is in line with the blood chemistry analysis. A summary of histopathological findings was included in the online supplementary materials. Note that the liver tissue specimen for pathological sectioning was collected postmortem ( 27 to 29 days after the challenge); by then the ALT level had already declined to (or slightly below) the normal range (Fig. 2E) and no viral RNA could be detected (Table 2). Not surprisingly, no viral antigen was found in the liver tissue by immunolabeling with the specific anti-S monoclonal antibody (data not shown). Therefore, it is likely that the liver inflammation shown in Fig. 3 does not reflect the true severity of the hepatitis associated with rMVA-S vaccination and SARS-CoV challenge and, in fact, may represent the recovering stage. Detailed pathological examination at the time when the ALT level is at the highest should be performed in future studies. Other organs were only mildly affected by SARS-CoV infection (data not shown).

It is known that neutralizing antibodies induced by the $\mathrm{S}$ protein of feline infectious peritonitis virus (also a coronavirus) often lead to accelerated infection by the mechanism of antibody-dependent enhancement of virus infectivity $(12,13,16$, 18). SARS-CoV has been shown to infect hepatocytes and cause hepatitis in humans (3). Here, we found that immunization with rMVA-S is associated with enhanced hepatitis in ferrets after SARS-CoV challenge. However, our present data cannot conclusively demonstrate whether or not the enhanced liver inflammation was the consequence of accelerated virus infection of livers or simply enhanced immunopathological effects on livers as a combined result from rMVA-S immunization and SARS-CoV infection. Further investigation, such as passive transfer of immune sera from vaccinated or SARS$\mathrm{CoV}$-infected ferrets to naive ferrets, which then are challenged with SARS-CoV, should aid in understanding the link between the immune responses induced by SARS-CoV antigens and enhanced liver inflammation.

In this communication, we demonstrated that vaccination with rMVA-S could induce a rapid and vigorous memory neutralizing antibody response, which is an essential feature for an effective prophylactic vaccine, in ferrets after challenge with SARS-CoV. On the other hand, our data suggest that vaccination with SARS-CoV S may lead to enhanced liver damage following SARS-CoV infection. This information is extremely important for development of safe SARS vaccines. Extra caution should be taken in proposed human trials of SARS vaccines (9) due to the potential liver damage from immunization and virus infection.

This work was supported by Health Canada and Canadian Food Inspection Agency.

We thank the continuous support from Frank Plummer, Paul Kitching, and Tim Booth. We also thank Mike Carpenter and Steven Jones for their critical reading of the manuscript and Peter Wright and Nicole Beausoleil for their help.

\section{REFERENCES}

1. Berry, J. D., S. Jones, M. A. Drebot, A. Andonov, M. Sabara, X. Y. Yuan, H. Weingartl, L. Fernando, P. Marszal, J. Gren, B. Nicolas, M. Andonova, F. Ranada, M. J. Gubbins, T. B. Ball, P. Kitching, Y. Li, A. Kabani, and F.
Plummer. 2004. Development and characterisation of neutralising monoclonal antibody to the SARS-coronavirus. J. Virol. Methods 120:87-96.

1a.Bisht, H., A. Roberts, L. Vogel, A. Bukreyev, P. L. Collins, B. R. Murphy, K. Subbarao, and B. Moss. 2004. Severe acute respiratory syndrome coronavirus spike protein expressed by attenuated vaccinia virus protectively immunizes mice. Proc. Natl. Acad. Sci. USA 101:6641-6646.

2. Chakrabarti, S., J. R. Sisler, and B. Moss. 1997. Compact, synthetic, vaccinia virus early/late promoter for protein expression. BioTechniques 23:1094-1097.

3. Chau, T. N., K. C. Lee, H. Yao, T. Y. Tsang, T. C. Chow, Y. C. Yeung, K. W. Choi, Y. K. Tso, T. Lau, S. T. Lai, and C. L. Lai. 2004. SARS-associated viral hepatitis caused by a novel coronavirus: report of three cases. Hepatology 39:302-310.

4. Collins, A. R., R. L. Knobler, H. Powell, and M. J. Buchmeier. 1982. Monoclonal antibodies to murine hepatitis virus-4 (strain JHM) define the viral glycoprotein responsible for attachment and cell-cell fusion. Virology 119: 358-371.

5. Falkner, F. G., and B. Moss. 1988. Escherichia coli gpt gene provides dominant selection for vaccinia virus open reading frame expression vectors. J. Virol. 62:1849-1854.

6. Fleming, J. O., S. A. Stohlman, R. C. Harmon, M. M. Lai, J. A. Frelinger, and L. P. Weiner. 1983. Antigenic relationships of murine coronaviruses: analysis using monoclonal antibodies to JHM (MHV-4) virus. Virology 131: 296-307.

7. Gao, W., A. Tamin, A. Soloff, L. D'Aiuto, E. Nwanegbo, P. D. Robbins, W. J. Bellini, S. Barratt-Boyes, and A. Gambotto. 2003. Effects of a SARS-associated coronavirus vaccine in monkeys. Lancet 362:1895-1896.

8. Marra, M. A., S. J. Jones, C. R. Astell, R. A. Holt, A. Brooks-Wilson, Y. S. Butterfield, J. Khattra, J. K. Asano, S. A. Barber, S. Y. Chan, A. Cloutier, S. M. Coughlin, D. Freeman, N. Girn, O. L. Griffith, S. R. Leach, M. Mayo, H. McDonald, S. B. Montgomery, P. K. Pandoh, A. S. Petrescu, A. G. Robertson, J. E. Schein, A. Siddiqui, D. E. Smailus, J. M. Stott, G. S. Yang, F. Plummer, A. Andonov, H. Artsob, N. Bastien, K. Bernard, T. F. Booth, D. Bowness, M. Czub, M. Drebot, L. Fernando, R. Flick, M. Garbutt, M. Gray, A. Grolla, S. Jones, H. Feldmann, A. Meyers, A. Kabani, Y. Li, S. Normand, U. Stroher, G. A. Tipples, S. Tyler, R. Vogrig, D. Ward, B. Watson, R. C. Brunham, M. Krajden, M. Petric, D. M. Skowronski, C. Upton, and R. L. Roper. 2003. The genome sequence of the SARS-associated coronavirus. Science 300:1399-1404.

9. Marshall, E., and M. Enserink. 2004. Medicine. Caution urged on SARS vaccines. Science 303:944-946.

10. Martina, B. E., B. L. Haagmans, T. Kuiken, R. A. Fouchier, G. F. Rimmelzwaan, G. Van Amerongen, J. S. Peiris, W. Lim, and A. D. Osterhaus. 2003 Virology: SARS virus infection of cats and ferrets. Nature 425:915.

11. Moss, B. 1996. Genetically engineered poxviruses for recombinant gene expression, vaccination, and safety. Proc. Natl. Acad. Sci. USA 93:11341-11348.

12. Olsen, C. W. 1993. A review of feline infectious peritonitis virus: molecular biology, immunopathogenesis, clinical aspects, and vaccination. Vet. Microbiol. 36:1-37.

13. Pedersen, N. C., and J. W. Black. 1983. Attempted immunization of cats against feline infectious peritonitis, using avirulent live virus or sublethal amounts of virulent virus. Am. J. Vet. Res. 44:229-234.

14. Rota, P. A., M. S. Oberste, S. S. Monroe, W. A. Nix, R. Campagnoli, J. P. Icenogle, S. Penaranda, B. Bankamp, K. Maher, M. H. Chen, S. Tong, A. Tamin, L. Lowe, M. Frace, J. L. DeRisi, Q. Chen, D. Wang, D. D. Erdman, T. C. Peret, C. Burns, T. G. Ksiazek, P. E. Rollin, A. Sanchez, S. Liffick, B. Holloway, J. Limor, K. McCaustland, M. Olsen-Rasmussen, R. Fouchier, S. Gunther, A. D. Osterhaus, C. Drosten, M. A. Pallansch, L. J. Anderson, and W. J. Bellini. 2003. Characterization of a novel coronavirus associated with severe acute respiratory syndrome. Science 300:1394-1399.

15. Subbarao, K., J. McAuliffe, L. Vogel, G. Fahle, S. Fischer, K. Tatti, M. Packard, W. J. Shieh, S. Zaki, and B. Murphy. 2004. Prior infection and passive transfer of neutralizing antibody prevent replication of severe acute respiratory syndrome coronavirus in the respiratory tract of mice. J. Virol. 78:3572-3577.

16. Vennema, H., R. J. de Groot, D. A. Harbour, M. Dalderup, T. GruffyddJones, M. C. Horzinek, and W. J. Spaan. 1990. Early death after feline infectious peritonitis virus challenge due to recombinant vaccinia virus immunization. J. Virol. 64:1407-1409.

17. Weingartl, H. M., J. Copps, M. A. Drebot, P. Marszal, G. Smith, J. Gren, M. Andova, J. Pasick, P. Kitching, and M. Czub. 2004. Susceptibility of pigs and chickens to SARS coronavirus. Emerg. Infect. Dis. 10:179-184.

18. Weiss, R. C., and F. W. Scott. 1981. Antibody-mediated enhancement of disease in feline infectious peritonitis: comparisons with dengue hemorrhagic fever. Comp. Immunol. Microbiol. Infect. Dis. 4:175-189.

19. Yang, Z. Y., W. P. Kong, Y. Huang, A. Roberts, B. R. Murphy, K. Subbarao, and G. J. Nabel. 2004. A DNA vaccine induces SARS coronavirus neutralization and protective immunity in mice. Nature 428:561-564. 